\title{
Convalescent plasma for adults with acute COVID-19 respiratory illness (CONCOR-1): study protocol for an international, multicentre, randomized, open-label trial
}

Philippe Bégin ${ }^{1,2}$, Jeannie Callum ${ }^{3,4}$, Nancy M. Heddle ${ }^{5,6}$, Richard Cook ${ }^{7}$, Michelle P. Zeller ${ }^{5,6,10}$, Alan Tinmouth 8,9,10, Dean A. Fergusson 11,12,13, Melissa M. Cushing ${ }^{14,15}$, Marshall J. Glesby ${ }^{16}$, Michaël Chassé ${ }^{17,18}$, Dana V. Devine ${ }^{10,19}$, Nancy Robitalle ${ }^{20,21}$, Renée Bazin ${ }^{22}$, Nadine Shehata ${ }^{23,24,25}$, Andrés Finzi ${ }^{26,27}$, Allison McGeer ${ }^{28,29}$, Damon C. Scales ${ }^{30,31}$, Lisa Schwartz ${ }^{32}$, Alexis F. Turgeon ${ }^{33,34}$, Ryan Zarychanski ${ }^{35}$, Nick Daneman ${ }^{36}$, Richard Carl ${ }^{5,6}$, Luiz Amorim ${ }^{37}$, Caroline Gabe ${ }^{6}$, Martin Ellis ${ }^{38,39}$, Bruce S. Sachais ${ }^{15,40}$, Kent Cadogan Loftsgard ${ }^{6}$, Erin Jamula ${ }^{6}$, Julie Carruthers ${ }^{6}$, Joanne Duncan ${ }^{6}$, Kayla Lucier ${ }^{6}, \mathrm{Na} \mathrm{Li}^{6,41,42}$, Yang Liu' ${ }^{6}$, Chantal Armali ${ }^{3}$, Amie Kron $^{3}$, Dimpy Modi ${ }^{3}$, Marie-Christine Auclair ${ }^{43}$, Sabrina Cerro ${ }^{43}$, Meda Avram ${ }^{43}$ and Donald M. Arnold ${ }^{5,6^{*}}$ (D)

\section{Abstract}

Background: Convalescent plasma has been used for numerous viral diseases including influenza, severe acute respiratory syndrome, Middle East respiratory syndrome and Ebola virus; however, evidence to support its use is weak. SARS-CoV-2 is a novel coronavirus responsible for the 2019 global pandemic of COVID-19 community acquired pneumonia. We have undertaken a randomized controlled trial to assess the efficacy and safety of COVID19 convalescent plasma (CCP) in patients with SARS-CoV-2 infection.

* Correspondence: arnold@mcmaster.ca

${ }^{5}$ Department of Medicine, McMaster University, Hamilton, Ontario, Canada ${ }^{6}$ McMaster Centre for Transfusion Research, McMaster University, Hamilton, Ontario, Canada

Full list of author information is available at the end of the article

C C The Author(s). 2021 Open Access This article is licensed under a Creative Commons Attribution 4.0 International License, which permits use, sharing, adaptation, distribution and reproduction in any medium or format, as long as you give appropriate credit to the original author(s) and the source, provide a link to the Creative Commons licence, and indicate if changes were made. The images or other third party material in this article are included in the article's Creative Commons licence, unless indicated otherwise in a credit line to the material. If material is not included in the article's Creative Commons licence and your intended use is not permitted by statutory regulation or exceeds the permitted use, you will need to obtain permission directly from the copyright holder. To view a copy of this licence, visit http://creativecommons.org/licenses/by/4.0/ The Creative Commons Public Domain Dedication waiver (http://creativecommons.org/publicdomain/zero/1.0/) applies to the data made available in this article, unless otherwise stated in a credit line to the data. 
(Continued from previous page)

Methods: CONCOR-1 is an open-label, multicentre, randomized trial. Inclusion criteria include the following: patients $\geq 16$ years, admitted to hospital with COVID-19 infection, receiving supplemental oxygen for respiratory complications of COVID-19, and availability of blood group compatible CCP. Exclusion criteria are : onset of respiratory symptoms more than 12 days prior to randomization, intubated or imminent plan for intubation, and previous severe reactions to plasma. Consenting patients are randomized 2:1 to receive either approximately 500 $\mathrm{mL}$ of CCP or standard of care. CCP is collected from donors who have recovered from COVID-19 and who have detectable anti-SARS-CoV-2 antibodies quantified serologically. The primary outcome is intubation or death at day 30. Secondary outcomes include ventilator-free days, length of stay in intensive care or hospital, transfusion reactions, serious adverse events, and reduction in SARS-CoV-2 viral load. Exploratory analyses include patients who received CCP containing high titre antibodies. A sample size of 1200 patients gives $80 \%$ power to detect a 25\% relative risk reduction assuming a $30 \%$ baseline risk of intubation or death at 30 days (two-sided test; $a=0.05$ ). An interim analysis and sample size re-estimation will be done by an unblinded independent biostatistician after primary outcome data are available for $50 \%$ of the target recruitment $(n=600)$.

Discussion: This trial will determine whether CCP will reduce intubation or death non-intubated adults with COVID-19. The trial will also provide information on the role of and thresholds for SARS-CoV-2 antibody titres and neutralization assays for donor qualification.

Trial registration: Clinicaltrials.gov NCT04348656. Registered on 16 April 2020.

Keywords: Convalescent plasma, SARS-CoV-2, Coronavirus, COVID-19, Randomized controlled trial

\section{Background}

COVID-19 is an acute viral illness caused by the novel SARS-CoV-2 coronavirus. With a high number of infections, therapeutic options are urgently needed to mitigate morbidity and mortality [1]. One potential therapeutic option is convalescent plasma collected from individuals who have recovered from COVID-19 [2]. COVID-19 convalescent plasma (CCP) contains antiSARS-CoV-2 antibodies, which can neutralize the virus, reduce viral load, and potentially improve clinical outcomes [3].

Convalescent plasma has been used during other pandemics. During the severe acute respiratory syndrome (SARS) epidemic, a non-randomized study from Hong Kong $(n=80)$ showed a reduction in mortality with convalescent plasma compared with historical controls $(13 \%$ vs. $17 \%$ respectively) [4]. For influenza, an observational study $(n=93)$ of convalescent plasma showed a reduction in mortality, with an odds ratio (OR) of 0.20 [95\% confidence interval (CI), 0.06-0.69] [5], and a phase 3 randomized trial $(n=200)$ using hyperimmune plasma did not show an improvement in clinical status on day 7 [6]. In Ebola, improved survival was observed in a nonrandomized study of convalescent whole blood [7], but not with convalescent plasma [8]. A systematic review of convalescent plasma for viral pneumonias caused by severe influenza or coronavirus infection (SARS-CoV-1 or Middle East respiratory syndrome (MERS)) included 32 non-randomized studies $(n=1327)$. The conclusion based on very low-quality evidence was that convalescent plasma may be associated with a reduction in mortality, length of stay, and duration of ventilation and that well-designed clinical trials are needed [9]. A recent systematic review that included four randomized controlled trials (RCTs) and two non-randomized studies of convalescent plasma or hyperimmune products showed lowquality evidence for lack of harm and insufficient evidence of efficacy [10].

Case series of CCP use in patients with COVID-19 from China reported positive results with CCP administered before 22 days of symptom onset [3, 11, 12]. Conversely, other studies reported no benefit when CCP was administered later in the course of disease ( $>21$ days) [13]. A randomized trial from China $(n=103)$ reported no improvement in outcomes with $\mathrm{CCP}$ although the direction of the effect suggested benefit for patients with severe but not life-threatening disease [14]. Another RCT $(n=86)$ from the Netherlands reported no improvement with CCP but the trial was stopped early when high titre anti-SARS-CoV2 antibodies were detected in patients at baseline [15]. An RCT from India $(n=464)$ showed no difference in mortality or disease progression, but CCP donors were not screened for antibody titres [16]. An RCT from Argentina $(n=228)$ showed no benefit of CCP compared with placebo for improvement in clinical status by day 30 (**:Please insert this new reference: Siminovich et al PMID: 33232588**), while another plaebo-controlled RCT from Argentina $(n=160)$ showed a reduction in the development of severe respiratory disease when high-titre CCP was given early in the disease course (within 72 hours of symptom onset) (*** Please insert this new reference: Libster et al PMID:33406353*) On March 25, 2020, the US FDA launched an Expanded Access Program for CCP [17]. 
An observational study of 35,322 patients who received CCP under that program suggested that CCP was most effective when given early in the disease course and with high titre anti-SARS-CoV2 antibodies [18]. A previous report of 20,000 transfused patients showed few adverse events with CCP $(<1 \%)$ such as allergic reactions, transfusion-associated circulatory overload (TACO), transfusion-related acute lung injury (TRALI), thromboembolic events, hypotension, or cardiac events [19, 20]. A theoretical risk of $\mathrm{CCP}$ is antibody-dependent enhancement of infection $[2,21]$.

\section{Methods/design \\ Objective}

CCP remains an unproven treatment for COVID-19. CONCOR-1 (CONvalescent Plasma for Hospitalized Adults with Acute COVID-19 Respiratory Illness) is a multicentre, open-label superiority trial designed to answer whether CCP, collected from individuals who have recovered from COVID-19 infection that contains antiSARS-CoV-2 antibodies, decreases the risk of intubation or death at day 30 in hospitalized adult patients with acute COVID-19 respiratory illness compared to standard of care. We hypothesize that CCP will reduce the risk of intubation and death at day 30 and improve other outcomes including the need for critical care support, duration of hospitalization, and quality of life. Results of serological testing on the CCP will be correlated with patient outcomes.

\section{Trial design}

CONCOR-1 is a parallel arm, multicentre, open-label, superiority randomized controlled trial comparing CCP to standard of care (Fig. 1). Centres in Canada, the
United States of America (USA), and Brazil are invited to participate. Eligible patients are randomized using a 2:1 allocation to receive either CCP or standard of care. The protocol was approved by all Research Ethics Boards at participating sites, the blood collection facilities, Health Canada (Control \# 238201), the United States Food and Drug Administration (IND 22075), and the National Research Ethics Commission (Comissão Nacional de Ética em Pesquisa) in Brazil (approval 4.305.792). The trial was registered at clinicaltrials.gov (NCT04348656).

\section{Study setting}

The trial will take place at approximately 80 academic and community hospitals in Canada, USA, and Brazil. A complete list of active study sites can be found in the trial record at clinicaltrials.gov.

\section{Eligible patients}

Patients are eligible for the trial if (1) age $\geq 16$ years; (2) hospitalized with confirmed SARS-CoV-2 infection; (3) receiving supplemental oxygen for COVID-19 respiratory illness; (4) ABO-compatible CCP is available. Exclusion criteria are : (1) onset of respiratory signs or symptoms $>12$ days before randomization; (2) currently intubated or a plan is in place for intubation; (3) a plan is in place for no active treatment; or (4) contraindication to plasma transfusion (e.g. history of anaphylaxis, refusal of blood products). These criteria are meant to capture patients who are early in the disease course and at risk of worsening respiratory illness. Co-enrolment in other interventional trials is permitted, with the exception of other CCP trials. We will collect data on all cointerventions and co-enrolment.

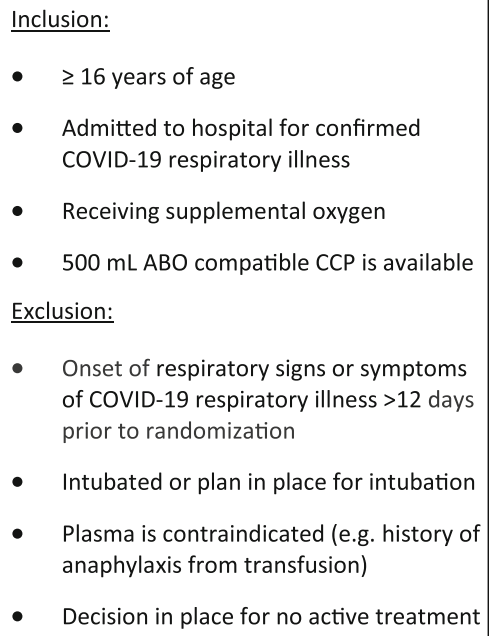

Fig. 1 Trial overview

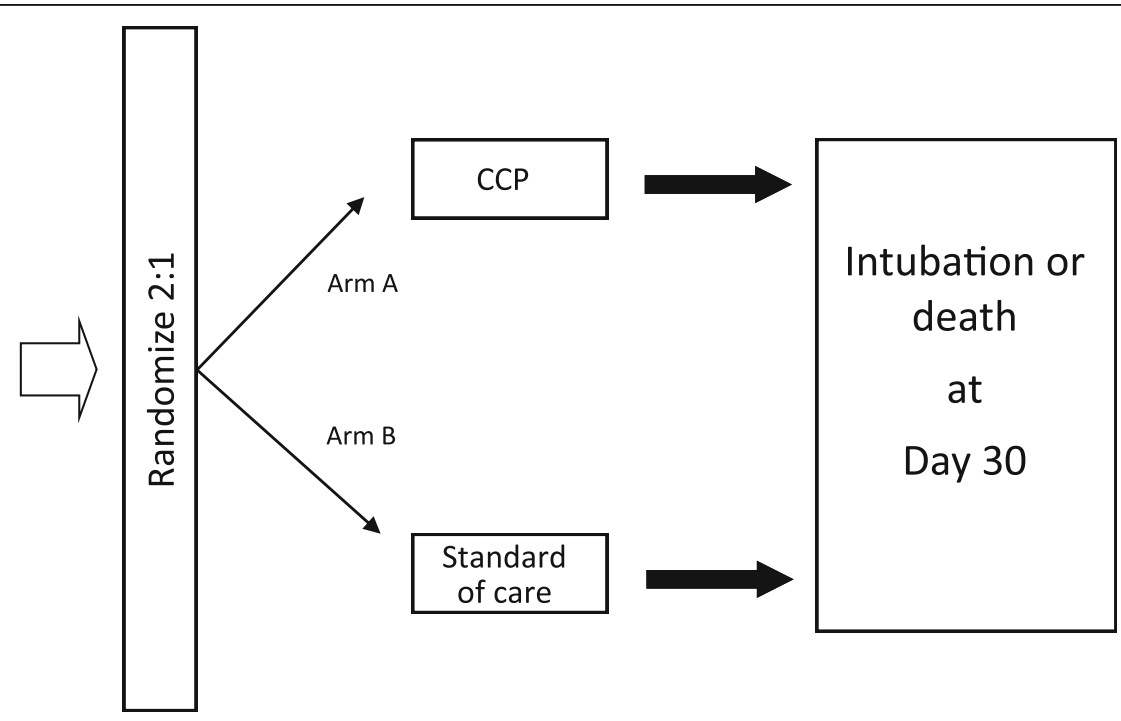




\section{Recruitment}

Sites work with their local infectious disease team to determine a strategy for identifying admitted patients who test positive for COVID-19 and to communicate these results to the research team. Infectious disease physicians act as site co-investigators wherever possible to facilitate this process. Sites submit screening data on a weekly basis to the methods centre, which include reasons for screen failures. Screening logs are reviewed regularly by the trial Executive Committee to identify common exclusions and strategies to optimize recruitment.

\section{Informed consent}

Informed consent is obtained from each participant by authorized study personnel prior to any study procedures taking place. Informed consent may be written or verbal. Obtaining verbal informed consent remotely is permitted to avoid exposing research staff to COVID-19 and may be facilitated by telephone or video-assisted consent tools. Where required by the local research ethics board, written informed consent is obtained from the patient or legally authorized representative who provided verbal consent, once safe to do so. Participants provide consent for use of blood samples for study testing as described in the protocol and for publication of the results using aggregate data. No additional biobanking is planned.

\section{Randomization}

Allocation sequence generation is performed by an independent biostatistician using a computer-generated algorithm and stratified by centre and age $(<60$ and $\geq 60$ years). Age is included as a stratum variable due to known increased mortality with age. The randomization sequence is generated using a secure, concealed, computer-generated system and blocked with random block sizes to ensure concealment. The allocation sequence is maintained securely within the electronic data capture software system and accessible only to the independent, unblinded statistician. Participants are randomized by authorized study personnel at each site who will assign participants to interventions using a centralized website. Access to this website is restricted to trained study individuals via a unique username and password.

\section{Intervention}

The experimental intervention is a single dose of approximately $500 \mathrm{~mL}$ of CCP. Apheresis CCP is will be collected by Canadian Blood Services (CBS), Héma-Québec (HQ), the New York Blood Center (NYBC), and Hemorio (Brazil). CCP donors must meet all donor eligibility criteria for routine apheresis plasma donation plus (1) confirmed COVID-19 infection by nasal swab or by antibody testing; (2) complete resolution of symptoms for at least 14 days; (3) male donors, or female with no pregnancy history or with a negative HLA antibody test result; and (4) $\geq 6$ days since last plasma donation. CCP will be compatible for $\mathrm{ABO}$ blood group or have evidence of low anti-A or anti-B titres $(<1: 50)$. CCP is e transfused by nursing staff as a single dose of approximately $500 \mathrm{~mL}$ from one donor, or two doses of $250 \mathrm{~mL}$ from one or two donors, administered within $24 \mathrm{~h}$ of randomization. The $500 \mathrm{~mL}$ dose of CCP is infused over $4 \mathrm{~h}$, and patients are monitored for adverse events during the infusion. This dose of CCP is consistent with previous CCP studies [3, 5, 22].

Anti-SARS-CoV-2 antibodies in donor plasma is measured by ELISA or cyclic enhanced immunofluorescence assay with a minimum titre of $1: 100$, or by neutralization assay with a minimum titre of $1: 160$. As there are no scientific data to support one qualifying antibody test over another, the blood suppliers were permitted to select the qualifying titre and methodology independently based on availability. The FDA has recommended the use of minimal titre of neutralizing antibodies of 1:160 (or 1:80 when no compatible units are available), recognizing that these thresholds lack validation and other biomarkers may be important (e.g. antibody-dependent cell cytotoxicity, complementmediated virolysis, or antibody-dependent presentation of antigens) (Fig. 2) [6, 23, 24]. At the end of the trial, all CPP units will be tested for neutralizing antibodies to correlate with clinical outcomes. Information collected on CCP donors includes donor age, date of onset and severity of COVID-19 symptoms, and SARS-CoV-2 antibody titres.

The control arm of the trial is standard of care. Standard of care treatment was not prescribed in the protocol. It consistsof supportive care treatments for respiratory illness, and specific treatments for COVID-19 available [25]. An open-label design was justified since the primary outcomes (intubation or death) are objective in nature. In addition, masking procedures such as plasma bag covers and additional labeling of plasma units would have imposed significant challenges to blood bank personnel during the pandemic, which would have have made the trial infeasible in many centres. The use of standard plasma as the control was not felt to be justified because of the potential harm with no anticipated benefit. The 2 : 1 allocation is anticipated to maximize acceptability by patients and local investigators and will improve power for sub-analyses of antibody titre.

Modifications to the intervention are not expected. Adherence to this one-time intervention will be monitored and is expected to be high; strategies to improve adherence will be developed if needed. The CCP 


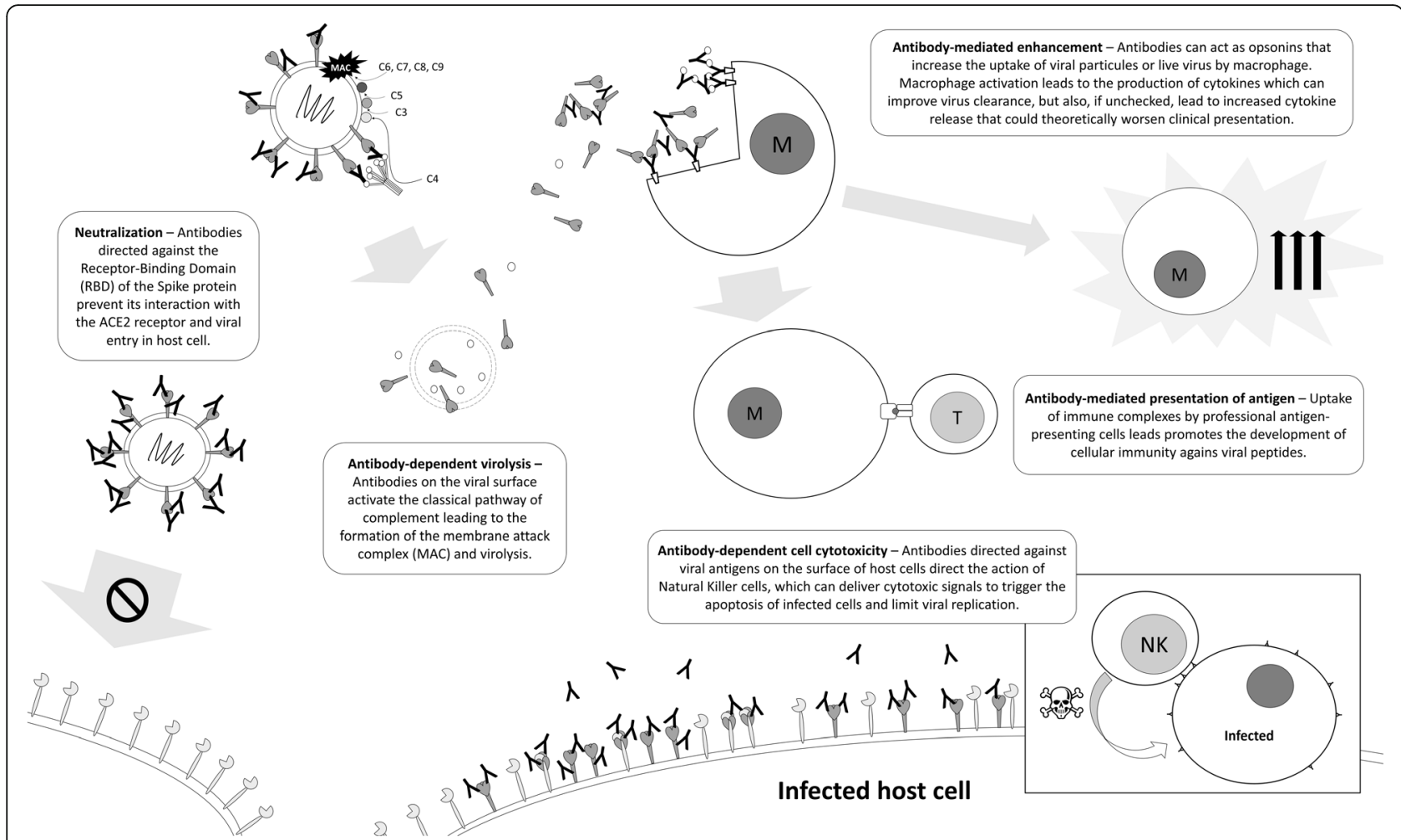

Fig. 2 Possible mechanisms of action of passively transferred antibodies in COVID-19. These include viral neutralization, complement-mediated antibody-dependent virolysis, antibody-dependent enhancement of immune response, antibody-mediated presentation of antigen, and antibodydependent cell cytotoxicity. Of these, only viral neutralization is measured by neutralization assays currently used to qualify convalescent plasma

infusion should bewill be stopped if an adverse event occurs that is deemed to be related to the CCP or at the patient's request. Discontinuation practices align with local clinical policies regarding management of transfusion reactions. Participants will receive routine clinical follow-up care as required for any harm caused by the trial.

All participants receive treatment as per local standard practice. In the rare event that a trial participant requires standard plasma for a non-study reason, local transfusion medicine labs will provide plasma with the longest storage duration (plasma is routinely stored for up to 1 year) to reduce of contamination with anti-SARSCoV-2 antibodies. All blood products received during the trial will be documented.

\section{Outcomes}

The primary outcome is a composite of intubation or death at day 30 . The primary outcome was chosen due to its clinical relevance and objective assessment. Secondary outcomes are time to intubation or death, ventilator-free days, in-hospital death by day 90 , time to in-hospital death, death by day 30, ICU length of stay, hospital length of stay, need for extracorporeal membrane oxygenation (ECMO), need for renal replacement therapy, myocarditis, patient-reported outcome as measured by EQ-5D-5 L, incremental cost per quality adjusted year, CCP transfusion-related adverse events and serious adverse events. Viral load from blood samples collected before and $48 \mathrm{~h}$ after randomization will be measured to determine the impact of CCP on viral clearance.

\section{Adverse event reporting and harms}

All transfusion-associated adverse events and adverse events with a severity of grade 3 or higher are captured. Adverse events are classified using the Medical Dictionary for Regulatory Activities (MedDRA) [26] and graded by the Common Terminology Criteria for Adverse Events (CTCAE) version 4.0 criteria [27]. Terminology and grading from the International Society on Blood Transfusion are recorded for transfusion-associated adverse events [28].

\section{Events requiring expedited reporting}

Suspected unexpected serious adverse reactions (SUSA $\mathrm{R})$ are reported to the sponsor within $24 \mathrm{~h}$. These are events that have a reasonable causal relationship to the CCP transfusion, are considered unexpected, and meet any of the following criteria for a serious adverse event: 
1. Results in death;

2. Is life-threatening; this means that the subject is at risk of death at the time of the event; it does not mean that the event hypothetically might have caused death if it was more severe;

3. Requires hospitalization (overnight or longer) or prolongation of existing hospitalization or invasive procedure;

4. Results in persistent or significant disability or incapacity;

5. Results in congenital anomaly or birth defect;

6. Is not be immediately life-threatening or result in death or hospitalization but may jeopardize the subject or require intervention to prevent one of the above outcomes.

Any TRALI or TACO event is reported to the sponsor within $24 \mathrm{~h}$.

Other unexpected serious adverse events which are not related to CCP are reported within $96 \mathrm{~h}$, if they meet reporting criteria. Due to the critical nature of illness of the study population, a revised definition of a reportable serious adverse event (SAE) was developed for use in this trial based off of guidelines used in critical care trials [29]. Therefore, the following events are not considered reportable SAEs for the purposes of the trial: AEs that are part of the natural history of the primary disease process or expected complications of critical illness; AEs expected in the context of a baseline medical condition; and AEs that are already captured as study outcomes. Figure 3 shows the flow diagram for adverse event reporting.

\section{Participant timeline}

Patients admitted to hospital with a positive COVID-19 test result are assessed for eligibility. Eligible patients who have consented are randomized as soon as possible (day of randomization is considered day 1). The assessment schedule for study patients is summarized in Table 1.

\section{Blinding}

CONCOR-1 is an open-label trial. The use of objective outcomes of death and intubation will mitigate bias as a result of the open-label design. The statistician performing the final analysis will remain blinded throughout the trial. Blinded safety reports will be generated at regular intervals for the Executive Committee, Independent Data Safety Monitoring Committee (IDSMC), and Steering Committee.

\section{Data collection}

Baseline, daily physiologic and laboratory data, and outcome data are collected from randomization to day 30
(Table 1). Data are entered into an electronic data capture system (EDC) by site personnel. The only required blood samples for the trial are the ABO blood group for determination of CCP compatibility and two samples for testing of viral load and antibody levels, which are sent to a central laboratory. Other laboratory tests are captured if ordered as part of routine patient care. All laboratory tests have been validated and undergo routine quality control as part of the clinical laboratories' quality management program.

Samples from all blood donors are tested for antibodies against SARS-CoV2 using quantitative and functional assays at centralized laboratories. No additional tests are planned for this trial.

Quality of life assessment is performed using the 5level EQ-5D instrument at baseline and at day 30 [30]. Data capture from electronic clinical charts and data entry are performed remotely wherever possible to mitigate infection risk to research personnel. Standardized training is provided to all site staff performing data collection and entry prior to site activation in the form of training videos, with proof of completion required to obtain a training certificate. An Operations Manual is provided to sites with detailed instructions for data collection and use of the forms within the EDC.

Participant retention and complete follow-up is not anticipated to be problematic as beyond day 1 (CCP infusion) and a follow-up contact at day 30, participants are not required to perform any additional trial-specific activities. The trial was designed to reduce burden on participants and promote retention. Participants who discontinue or deviate from the study protocol will still have all study data collected, unless they withdraw consent for study participation.

\section{Data management}

The CONCOR-1 database and randomization platform utilizes the REDCap (Research Electronic Data Capture) software, located on a secure authenticated server at the CONCOR-1 methods centre, McMaster University Medical Centre, Hamilton, Ontario, Canada [31, 32]. REDCap is a secure, web-based software platform designed to support data capture for research studies. Study patient data may be initially collected on paper CRFs (Additional file 1) and consent and eligibility confirmed before being entered into the eCRF. Upwards of 60 hospitals (or hospital groups) may participate, each able to input and access their own site data. The responsibilities of the sites for collection and inputting of data, timeliness, and responding to data queries are outlined in the Operations Manual. A comprehensive description of the longitudinal design of the trial, site eCRF training, electronic query/resolution workflow for ongoing quality review (based on documented data cleaning rules and 


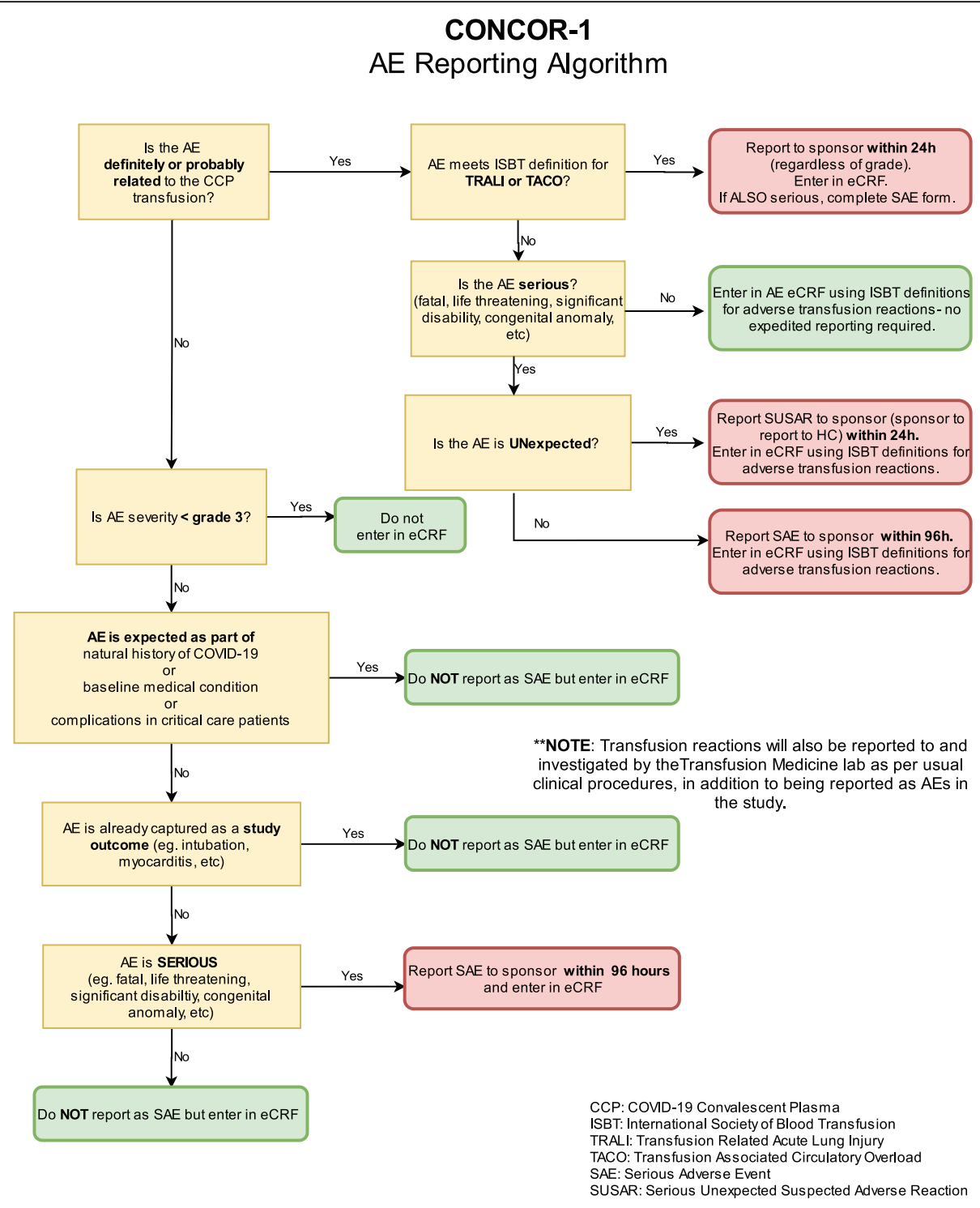

Fig. 3 Flow chart for AE and SAE reporting in the trial

validation rules), and access to data are documented in the CONCOR-1 Data Management Plan. All paper CRFs are found in the REDCap database file repository folder for easy download by sites, along with a link to all trial procedures and protocols.

\section{Sample size estimation}

We estimate that 1200 patients will be required to achieve $80 \%$ power to detect a relative risk reduction of $25 \%$ with CCP (2-tailed test at level $\alpha=0.05$ and 2:1 allocation), assuming a $30 \%$ baseline risk of intubation or death at day 30 with standard of care. This standard of care event rate estimate was derived from Canadian data where the risk of ICU admission among hospitalized patients was 23\% [33], and data from New York City where up to $41.5 \%$ of hospitalized patients required mechanical ventilation [34]. Because of uncertainty of baseline event rate, a sample size re-estimation is planned for when the primary outcome is available for $50 \%$ of the target sample. Sample size can be adjusted upwards if the observed event rate suggests that the trial is underpowered for the test based on the primary outcome.

\section{Statistical analysis plan}

The statistical analysis plan is provided in Additional file 2. A single interim analysis is planned when 
Table 1 Schedule of patient assessments

\begin{tabular}{|c|c|c|c|c|}
\hline \multirow[b]{2}{*}{ Visit } & \multirow[b]{2}{*}{ Screening } & \multirow[b]{2}{*}{ Baseline (D1) } & \multicolumn{2}{|l|}{ Follow-up } \\
\hline & & & $\begin{array}{l}\mathrm{D} 2-\mathrm{D} 30^{(1)} \\
\text { (or until discharge) }\end{array}$ & $\begin{array}{l}\mathrm{D} 30^{(2)} \\
\text { (if patients discharged }^{(10)}\end{array}$ \\
\hline Time window (days) & & & & $+/-3$ days \\
\hline Informed consent/assent & $x$ & & & \\
\hline Verify eligibility criteria & $x$ & & & \\
\hline Randomization & & $x$ & & \\
\hline CCP administration ${ }^{(3)}$ & & $x$ & & \\
\hline Demographics & & $x$ & & \\
\hline Comorbidities & & $x$ & & \\
\hline COVID-19 history ${ }^{(4)}$ & & $x$ & & \\
\hline Height and weight & & $x$ & & \\
\hline Vital signs & & $x$ & & \\
\hline $\mathrm{SpO}_{2}$ & & $x$ & $x$ & \\
\hline $\mathrm{FiO}_{2}$ & & $x$ & $x$ & \\
\hline COVID treatments & & $x$ & $x$ & $x$ \\
\hline Hospitalization daily assessment ${ }^{(5)}$ & & $x$ & $x$ & \\
\hline Results of blood tests (done as per clinical need) & & $x$ & $x^{(6)}$ & \\
\hline$E Q-5 D^{(7)}$ & & $x$ & & $x$ \\
\hline Vital status (dead/ alive) & & & $x$ & $x$ \\
\hline Adverse event review & & $x$ & $x$ & $x$ \\
\hline Discharge status ${ }^{(8)}$ & & & & $x$ \\
\hline Mechanical ventilation since discharge & & & & $x$ \\
\hline Viral load, cytokines and anti-CoV-2 titres $^{(9)}$ & & $x$ & $x$ & \\
\hline
\end{tabular}

(1) Only collected if still hospitalized

(2) Phone call if subject is already discharged from the hospital

(3) Only for subject randomized to the CCP treatment

(4) Includes date of first symptoms; description of symptoms and date of diagnostic test

(5) Mechanical ventilation, ECMO, renal replacement therapy, or myocarditis

(6) Daily from day 1 to 7 , then day $14 \pm 3$ days

(7) EQ-5D to be collected on day 30 regardless of hospitalization status

(8) Discharge or hospital status (admitted, home, local hospital, long-term care facility)

(9) To be performed using frozen leftover sample or extra tube collected once at baseline and once during follow-up between D2 and D6

(10) For patients still hospitalized at day 30, chart review will be conducted at day 90 to determine whether the patient was discharged or died in hospital between day 30 and day 90 or is still in hospital at day 90

data on the primary outcome are available for $50 \%$ of the target sample. The Lan-DeMets spending function [35] analog of the O'Brien-Fleming stopping rule [36] will be used to monitor the primary endpoint to guide the IDSMC. Conditional power will be presented for futility analysis as additional information for the IDSMC. A recommendation for premature trial termination may be made if there is reasonable cause, including but not limited to (1) a concern regarding an unacceptable risk to subjects, (2) results of the interim analysis demonstrating superiority, (3) results of the interim analysis for the revised sample size making the resultant trial infeasible due to the large number of patients required, (4) evidence from the futility analysis is compelling, (5) overall compliance with the protocol is poor, (6) data that are not sufficiently complete and/or are not evaluable, (7) regulatory authorities recommend termination of the trial, or (8) novel scientific data on the efficacy or safety of CCP becomes available raising concerns about the safety of CCP.

The primary outcome will be analysed using a 2sided Wald test of the null hypothesis that probability of intubation or death at day 30 is the same among individuals receiving $\mathrm{CCP}$ or standard of care with a relative risk and an associated 95\% confidence interval presented. A secondary analysis of time to event responses will be modeled using cause-specific Cox regression models accommodating censoring at the time of study withdrawal or upon the occurrence of competing events.

For secondary outcomes the restricted mean number of ventilator-free days will be compared using a 
nonparametric analysis. The survival status at day 30 and day 90 will be compared based on a comparison of binomial proportions, and the proportion of patients needing ECMO, needing renal replacement therapy, and/or with myocarditis will be compared between the two arms. Subgroup analyses will include:

- Co-enrolment in other therapeutic trials

- Age $(\geq 60)$

- Sex

- Ethnicity

- Obesity

- Medical comorbidities (diabetes, cardiac, respiratory)

- Smoking status (> 15 pack year history; never/ever/ current smoker)

- Number of CCP donors (one vs. two)

- Severity of illness in donors (hospitalized vs. not)

- Onset of any symptom (> 12 days vs. $\leq 12$ days)

- Timing of administration of CCP from diagnosis ( $\leq$ $72 \mathrm{~h}$ vs. $>72 \mathrm{~h}$ )

- $\mathrm{ABO}$ blood type of recipient

- Detectable vs non-detectable viral plasma viral load at baseline

- Anti-SARS-CoV-2 seropositivity at baseline

- Oxygen status at baseline ( $\leq 2 \mathrm{~L}$ vs $>2 \mathrm{~L}$ )

We expect to have complete data on individuals for the primary and secondary outcomes and we will make all efforts to minimize loss to follow-up. If an individual withdraws from the trial early, their time to event outcomes will be censored at the time of withdrawal. For binary outcomes where data are missing, the first analysis will be based on a completed cases (modified intention to treat) sample. A second analysis will deal with potential bias from using a complete case analysis by imputing missing data via imputation models fitted to the data from individuals providing complete information. Major protocol deviations and violations will be entered in the EDC in real time and monitored monthly throughout the trial by a subgroup of individuals from the Executive Committee and corrective action taken whenever possible. The Statistical Analysis Plan provides details related to handling of missing data and nonadherence to the protocol.

\section{Confidentiality}

No identifying information is collected until patients consent to trial participation. If allowed by local ethics boards, screening logs may contain patient initials but this information remains at the study site. For enrolled participants, case report forms (both paper and electronic) are identified by a unique study identification number. The study key containing the study identification number and patient identifiers will be securely stored at each study site. Any source documentation required to be sent off-site will be deidentified prior to transmission. Sites will store all study records securely, with access restricted to authorized individuals, for the duration of the trial and required retention period.

\section{Data monitoring}

The IDSMC is comprised of six individuals with expertise in biostatistics, transfusion medicine, infectious disease, and clinical research methodology (see IDSMC charter- Additional file 3). The first official meeting is planned for when the day 30 follow-up is completed for the first 20 randomized patients. Report formats will be reviewed and approved by the IDSMC. After the initial meeting, the IDSMC will meet when complete data is available for every 100 patients enrolled to review operational and safety data (see "Adverse event reporting and harms" section). The IDSMC will also receive monthly safety reports. The IDSMC will report to the study sponsor and the study Executive Committee, which includes the co-principal investigators.

The interim analysis is described in the Statistical Analysis Plan section. The results of the interim analysis will be unblinded for the independent biostatistician performing the analysis and the members of the IDSMC. All other study personnel and investigators will remain blinded to the results of the interim analysis. The primary purpose of the interim analysis is for sample size re-estimation. An O'Brien-Fleming stopping rule [36] will be used at that time, but treated as a guideline, so there is minimal impact on the threshold for statistical significance for the final significance test of the primary outcome. The IDSMC can make recommendations related to stopping for efficacy, futility, and/or safety, but the Steering Committee will be responsible for making the final decision.

\section{Auditing}

An independent third-party auditor facilitates site activation, verifies accuracy of data entered into the electronic case report form, and oversees GCP compliance on behalf of the sponsor. A detailed auditing plan describes the procedures and frequency of audits and the documents to be reviewed. Visits may be performed remotely as required by pandemic restrictions, or may take place on-site. In the event of a remote auditing visit, deidentified source documentation will be provided to the auditors. Audit reports will be provided to the sponsor after each visit. Visits will be performed after the first patient has been enrolled at a site, and regularly thereafter once a certain number of patients have subsequently been enrolled. 


\section{Trial management}

\section{Methods centre and coordinating centres}

The methods centre for the trial is the McMaster Centre for Transfusion Research, McMaster University. The methods centre will work directly with the blood suppliers to ensure the effective operations of all aspects of the trial. The methods centre is responsible for regulatory requirements, data management, and data analysis and oversees the working groups for CCP distribution and communications, as well as regulatory affairs, site activation, and blood banks outside Québec. The logistics centre (University of Toronto, QUEST research team) is responsible for developing study procedures, site blood bank and clinical team training modules, site support, and site recruitment outside Québec. The Québec coordinating centre (Ste-Justine, Université de Montréal) is responsible for site recruitment, training and activation, regulatory affairs, and blood banks in Québec, as well as serological analyses. The New York coordinating centre (Weill Cornell Medicine) will work with NYBC (supplier of CCP) and is responsible for regulatory requirements in the USA and for the coordination of the three New York hospital sites. Hemorio will act as the coordinating centre in Brazil and will oversee CCP supply in that country. Each coordinating centre includes physicians with transfusion medicine and/or immunological expertise, methodologists, experienced research coordinators, and research and administrative support staff (Fig. 4).

\section{Trial oversight}

The Steering Committee (SC) has the overall responsibility for the design, execution, analysis interpretation, and publication of results of the trial. The SC includes the broad range of clinical expertise from haematology, immunology, virology, critical care, research methodology, transfusion medicine required for the conduct of this large-scale RCT, blood supplier representatives, and a patient advisor.

SC members, affiliations, and areas of expertise are as follows:

- Dr. Donald Arnold; Department of Medicine, McMaster University; Hamilton, Ontario, Canada (haematology; transfusion medicine)

- Dr. Philippe Bégin; Department of Pediatrics, CHU Sainte-Justine; Department of Medicine, CHUM,

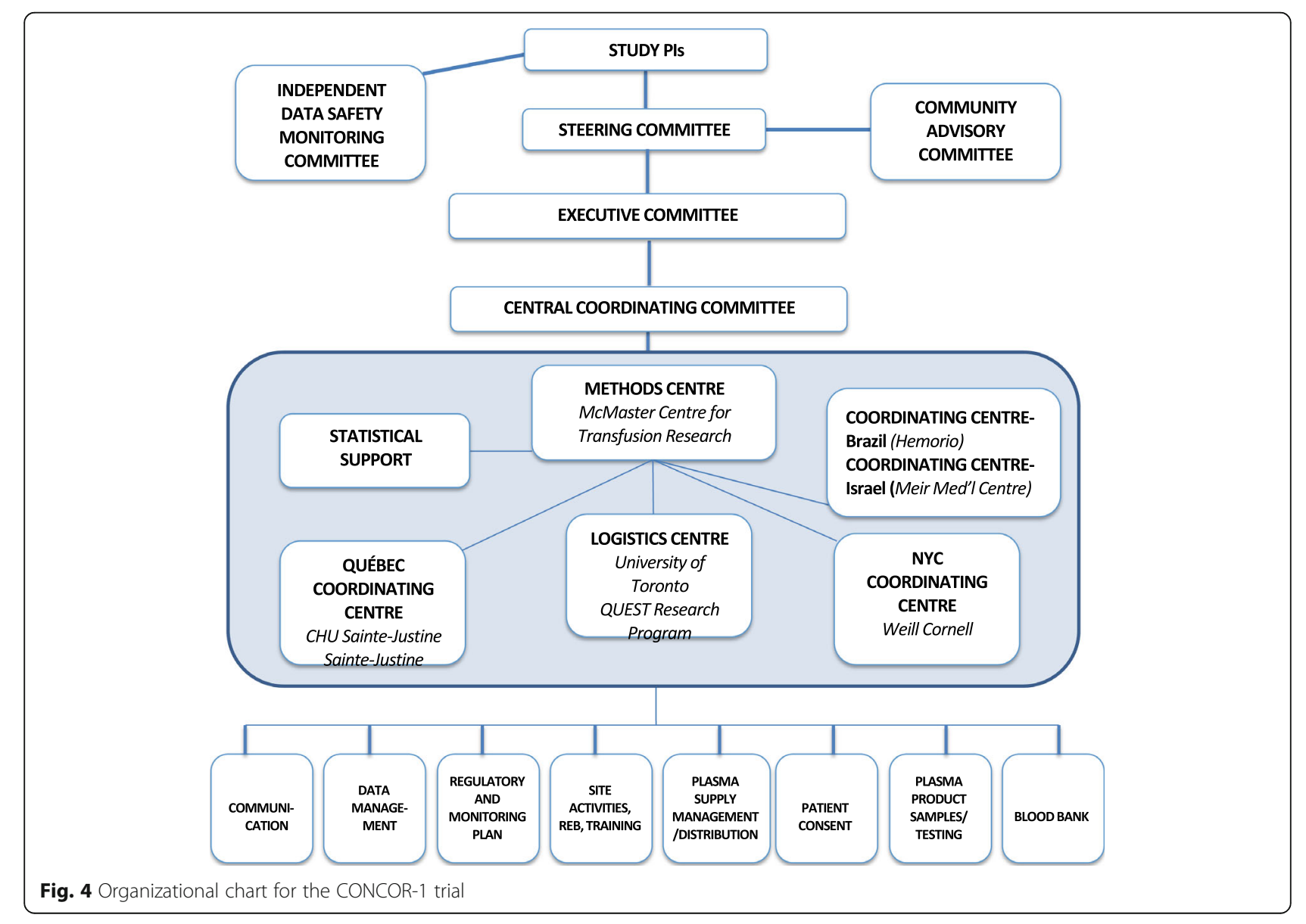


Université de Montréal; Montréal, Québec, Canada (immunology)

- Dr. Jeannie Callum; Department of Laboratory Medicine and Molecular Diagnostics, Sunnybrook Health Sciences Centre; Department of Laboratory Medicine and Pathobiology, University of Toronto; Toronto, Ontario, Canada (transfusion medicine)

- Prof Nancy Heddle; Department of Medicine, McMaster University; Hamilton, Ontario, Canada (transfusion medicine, clinical trial methodology)

- Dr. Richard Cook; Department of Statistics and Actuarial Science, University of Waterloo; Waterloo, Ontario, Canada (statistics; clinical trial design and analysis)

- Dr. Michelle Zeller; Canadian Blood Services; Department of Medicine, McMaster University; Hamilton, Ontario, Canada (transfusion medicine)

- Dr. Alan Tinmouth; Department of Medicine, University of Ottawa; Ottawa Hospital Research Institute; Canadian Blood Services, Ottawa, Ontario, Canada (haematology, transfusion medicine)

- Dr. Dean Fergusson; Ottawa Hospital Research Institute; Department of Medicine, University of Ottawa; Canadian Blood Services (clinical trial methodology, knowledge translation, transfusion medicine)

- Dr. Melissa Cushing; Transfusion Medicine and Cellular Therapy, NewYork-Presbyterian; Department of Pathology and Laboratory Medicine, Weill Cornell Medicine; New York City and Ithaca, New York, USA (transfusion medicine)

- Dr. Michaël Chassé; Department of Medicine (Critical Care), University of Montreal Health Centre (CHUM); Department of Medicine, University of Montreal; Montréal, Québec, Canada (critical care, clinical trial methodology)

- Dr. Dana Devine; Canadian Blood Services; Department of Pathology and Laboratory Medicine, University of British Columbia; Vancouver, British Columbia, Canada (transfusion medicine)

- Dr. Nancy Robitaille; Héma-Québec; Department of Pediatrics, CHU Sainte-Justine, Université de Montréal; Montréal, Québec, Canada (transfusion medicine, haematology)

- Dr. Renée Bazin; Medical Affairs and Innovation, Héma-Québec, Québec City, Québec, Canada (transfusion medicine, immunology)

- Dr. Nadine Shehata; Departments of Medicine, Laboratory Medicine and Pathobiology, Institute of Health Policy Management and Evaluation, University of Toronto; Division of Hematology, Mount Sinai Hospital; Canadian Blood Services, Toronto; Toronto, Ontario, Canada (transfusion medicine)
- Dr. Damon Scales; Department of Critical Care Medicine, Sunnybrook Health Sciences Centre; Department of Medicine, University of Toronto; Toronto, Ontario, Canada (critical care)

- Dr. Allison McGeer; Department of Microbiology, Sinai Health System; Department of Laboratory Medicine and Pathobiology and Dalla Lana School of Public Health, University of Toronto; Toronto, Ontario, Canada (infectious disease)

- Dr. Alexis Turgeon; Department of Anesthesiology and Critical Care Medicine, Division of Critical Care Medicine, Faculty of Medicine, Université Laval; CHU de Québec - Université Laval Research Centre, Population Health and Optimal Health Practices Research Unit, Trauma - Emergency Critical Care Medicine, Université Laval; Québec City, Québec, Canada (critical care)

- Dr. Lisa Schwartz; Department of Health Research Methods, Evidence \& Impact, McMaster University; Hamilton, Ontario, Canada (bioethics)

- Dr. Ryan Zarychanski; Department of Internal Medicine, University of Manitoba; Winnipeg, Manitoba, Canada (haematology, critical care)

- Dr. Nick Daneman; Department of Medicine, Sunnybrook Health Sciences Centre, University of Toronto; Toronto, Ontario, Canada (infectious disease, health policy)

- Dr. Luiz Amorim; Hemorio, Rio de Janeiro, Brazil (transfusion medicine, haematology)

- Dr. Bruce Sachais; New York Blood Center Enterprises, New York, NY; Department of Laboratory Medicine and Pathobiology, Weill Cornell Medical College; New York City, New York, USA (transfusion and apheresis medicine) Mr. Richard Carl (patient representative)

- Mr. Kent Cadogan Loftsgard (community advisor, specialty support to Recruitment Communications)

\section{Executive committee}

The Executive Committee (EC) oversees the day to day operations of the trial including monitoring trial progress, approving inter-institutional agreements, data management, and quality assurance. Members include the following: Dr. Donald Arnold, Dr. Philippe Bégin, Dr. Jeannie Callum, Prof Nancy Heddle, Dr. Alan Tinmouth, Dr. Michelle Zeller, Dr. Marshall Glesby, Dr. Melissa Cushing, and Dr. Richard Cook. Ad hoc members are Dr. Ryan Zarychanski, Dr. Nancy Robitaille, Dr. Dana Devine, and Dr. Alexis Turgeon.

\section{Independent data safety monitoring committee (IDSMC)}

The IDSMC includes members from both Canada and the USA. These individuals have expertise in transfusion medicine, infectious disease, cardiology, clinical trials, 
methodology, epidemiology, and biostatistics. An independent biostatistician will provide regular reports to the IDSMC. The IDSMC will monitor the safety and protection of human subjects participating in the trial; the proper conduct of the trial; and the ongoing scientific integrity, validity, and clinical and scientific relevance. The IDSMC will provide recommendations about continuing, modifying, and/or stopping the trial based on considerations of treatment outcomes, patient safety, and trial futility as appropriate.

\section{Communications}

As the structure of the CONCOR-1 trial evolved, an organizational chart (Fig. 4) was created to optimize flow of information to all relevant groups. Any protocol amendment will first require approval by the relevant national regulatory bodies, then the methods centre will be responsible for communicating protocol amendments to the study sites (in the form of new versions of the protocol and a summary of changes document) and collecting copies of the necessary local approvals prior to implementation.

\section{Access to data}

Study data may be made available to other investigators upon request. Such requests must first be approved by the Steering Committee.

\section{Dissemination plans}

The principal investigators will be responsible for publication of the data (manuscripts, abstracts, and posters, etc.) and are committed to publish and disseminate the study results in a timely manner without excessive restriction, regardless of whether the initial study hypothesis is confirmed or not. Investigators will not publish any data without approval from the Steering Committee. Medical writers will not be used. Authorship eligibility will comply with the International Committee of Medical Journal Editors criteria. The CONCOR-1 protocol has already been shared with US and European coalitions to allow investigators to collaborate on a core protocol and to share data. Results will be disseminated to patients and the public through the CONCOR-1 website (https://concor1.ca/), the media, and to various community groups identified by the study's Community Advisory Committee members. Site investigators will also play a role in distributing results to patients and colleagues.

\section{Ancillary and post-trial care}

There is no specific ancillary or post-trial care related to the trial; however, patients will continue to receive standard of care for their underlying condition as per clinical practice. Insurance to cover the cost of compensation for participants who suffer harm from trial participation is available for Canadian and American participants.

\section{Discussion}

Initial discussions to establish a Canadian trial of CCP began in late March 2020. The first CCP unit was collected 1 month later on April 24, 2020. The first patient was randomized on May 14, 2020. Rapid and intense coordination included the assembly of the Steering Committee with the range of required expertise, and coordination with numerous hospital sites and the blood suppliers. The organizational structure for the trial evolved quickly to define the roles of the methods centre, coordinating centres, and trial-specific working groups to allow for broader distribution of the trial workload to achieve trial launch at a rapid pace.

Given the context of the pandemic, there was no opportunity for a pilot study; nevertheless, we utilized a large, experienced and diverse Steering Committee to ensure as many challenges as possible will be avoided though strategic planning. Access to plasma donors was enhanced through public awareness campaigns, partnerships with public health, and an increasing number of recovered patients. To maximize access to the trial and enhance recruitment, we engaged approximately 50 Canadian centres, 3 hospitals in New York City (Weill Cornell Medicine), and centres in Brazil with a contingency plan to add more sites if needed. With this extensive recruitment plan, we hope to achieve our recruitment target within approximately 18 months. The eligibility criteria and outcomes in CONCOR-1 are harmonized with CCP trials in other countries. We will make our protocol publicly available through consortia in the USA and to allow investigators to share data and ensure completeness if local outbreaks taper off before sites are able to enroll their target sample size.

\section{Modeling supply and demand in an evolving pandemic}

The success of the trial depends on both the supply of CCP from individuals who have recovered from COVID-19 and the demand from eligible patients with acute COVID-19 infection. Factors affecting supply include the number of recovered patients with adequate SARS-CoV-2 antibody titres, willingness of donors to provide CCP, validation of SARS-CoV-2 antibody testing (ELISA and neutralization assays), and turnaround time for antibody testing. Factors affecting patient recruitment are the number of new admissions for COVID-19 infections at participating sites, severity of COVID-19 infections for admitted patients, and speed of activation of study sites. Many of these factors were not known when the protocol was designed, but the investigators and Steering Committee prioritized the development of a 
rigorous protocol that could be implemented quickly. We modeled supply of CCP and demand of COVID-19 patients in Quebec based on information available at the time of protocol development. We partnered with hospitals in New York City and Brazil to maximize recruitment. In addition, our endpoint is harmonized with other CCP trials internationally.

We chose to study hospitalized patients with respiratory compromise within the first 12 days of respiratory symptom onset as this patient population appeared to experience the greatest benefit from convalescent plasma [9]. As passive immunization may be more effective early in the course of illness before high enough levels of antibodies are formed, patients with onset of respiratory symptoms more than 12 days from potential randomization are not eligible for participation. For patients with more advanced disease (e.g. critically ill requiring invasive mechanical ventilation), the benefits of CCP appear to be less evident $[14,37]$.

The primary outcome is the composite of intubation or death at day 30 . Intubation is a clinically relevant outcome because of the high proportion of patients who require intubation (up to 40\%) [14], the association with intubation and mortality, and the desire to demonstrate a reduction in healthcare utilization. By introducing mortality via the composite outcome, we address situations in which intubation cannot be offered due to patient or physician preference or lack of availability of ventilators. The pattern of intubation practices during the pandemic and the availability of ventilators and intensive care beds were assessed when the protocol was designed through an informal survey of intensive care physicians. We recognized that patterns of practice are dynamic and could evolve over the epidemic. Due to the uncertainty around the $30 \%$ baseline estimate of the event rate, we included an adaptive sample size recalculation mid-way through the trial so that the sample size can be adjusted if appropriate.

\section{Equitable CCP distribution}

As part of the CONCOR-1 trial, we have established a platform for the distribution of CCP to ensure equity of plasma availability to hospitals and patients throughout Canada and the NYC sites while balancing optimal patient recruitment. This platform allows research staff to reserve an $\mathrm{ABO}$-compatible unit for an eligible patient and release it back into inventory if the patient is randomized to standard of care. We developed a distribution model where CBS provides CCP to hub hospitals that distribute to other proximal sites. This CCP distribution platform is shared with other CCP trials in Canada for critically ill patients (REMAP-CAP: NCT02735707) and pediatrics (CONCOR-Kids: NCT04377568). The CCP distribution working group consists of representatives from all Canadian CCP trials, the blood suppliers, hospital transfusion services, a bioethicist, an operations researcher who developed the software application and a full-time research coordinator who will monitor CCP inventory daily. For sites in Québec, New York, and Brazil, the blood suppliers will distribute the CCP directly to the participating sites. Agreements between HQ and CBS will facilitate sharing of $\mathrm{CCP}$ for less common blood groups (B and $\mathrm{AB}$ ).

The protocol was designed to mitigate risk to research and clinical staff during the pandemic by including remote processes for consent, data collection, and monitoring that do not rely on in-person contact. Data collection will be done through a daily review of the health record and telephone contact with the clinical team as required, and data monitoring will be done by remote access to health records until on-site monitoring is permitted. No additional procedures are required for the trial except for the CCP transfusion and a screening $\mathrm{ABO}$ blood group, which may be routine for all COVID19 patients in certain study sites. Both study samples (baseline and $48 \mathrm{~h}$ after randomization) for viral load and antibody levels can be collected at the time of a preplanned blood draw or from sample discards from the clinical laboratories.

Co-enrolment in drug trials is permitted. This will require collaboration with other site investigators to harmonize screening and consent procedures and establish trial prioritization. The rate of co-enrolment will be prospectively monitored by the IDSMC and the SC and the effect of co-enrolment will be evaluated in subgroup analyses. We anticipate that the rate of co-enrolment will be less than $20 \%$ since CONCOR-1 will recruit from many community hospitals where other COVID-19 trials are not open.

\section{Serology}

Antibody testing to qualify $\mathrm{CCP}$ products is done by ELISA, cyclic enhanced immunofluorescence assay, or viral neutralization assays. This strategy is aligned with FDA recommendations on the use of CCP [38] and accommodates the differences in the donor qualification requirements by the blood suppliers. As a secondary analysis, we will explore the relation between clinical outcomes and CCP anti-receptor-binding domain (RBD) and neutralizing antibody titres, which will be ultimately measured in all units. A previous non-randomized retrospective study reported that patient receiving a single unit of CCP with an anti-SARS-CoV2 titre greater than $18.35 \mathrm{~s} / \mathrm{co}$ had lower mortality rate compared to those receiving one CCP unit with a titre lower than $4.6 \mathrm{~s} / \mathrm{co}$ (Ortho VITROS IgG assay) (unpublished pre-print). Figure 5 shows how these dosages compare in terms of final amount of anti-SARS-CoV2 antibody transfused based 


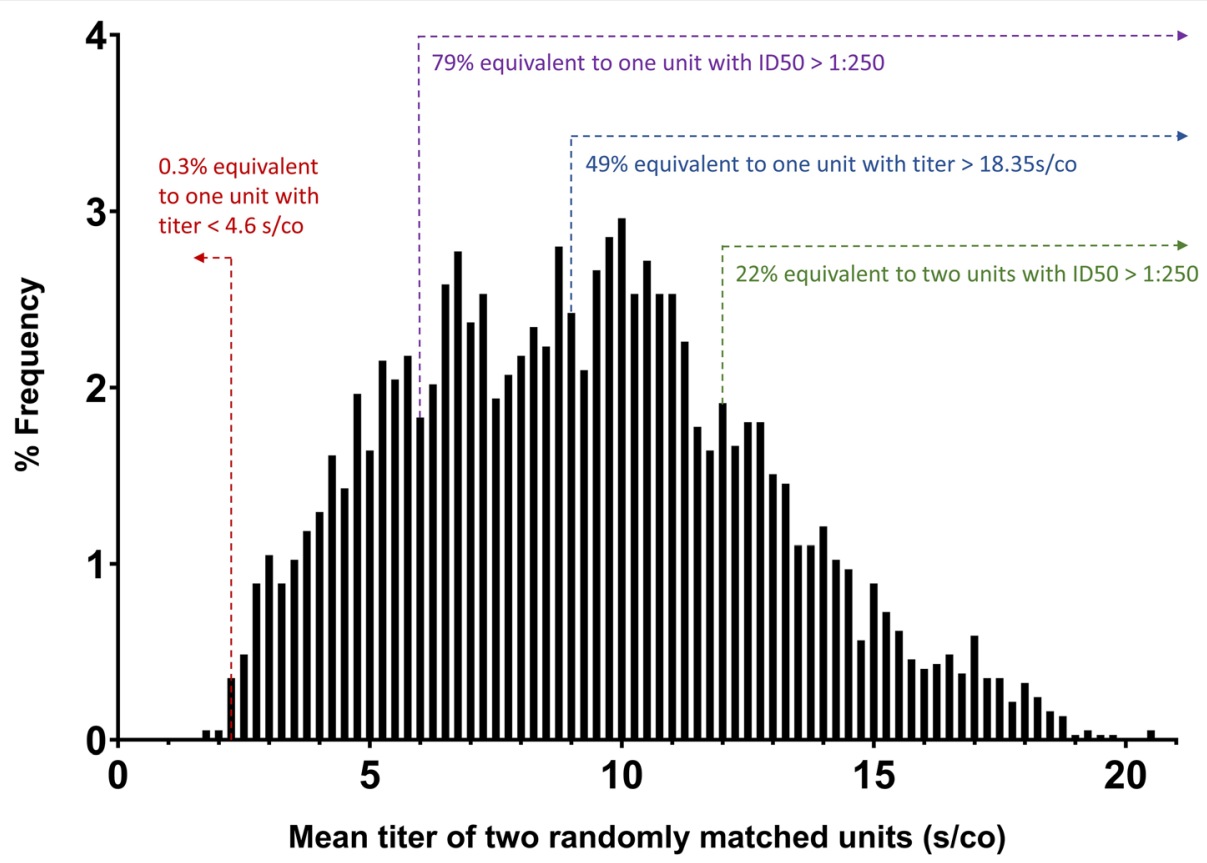

Fig. 5 Expected distribution of transfused plasma potency. The distribution of mean antibody titre in transfused convalescent plasma units was projected by performing a simulation in which plasma units from different donors were paired randomly. The simulation is based on the titres of 1150 plasma units currently in inventory at one of the blood suppliers (Héma-Québec). Titers were originally established with an in-house ELISA but have been translated in equivalent d/co units from the ORTHO VITRIOS IgG assay

on a simulation based of CCP collected to date for the trial at the HQ sites, and assuming transfusion of two units from randomly matched donors. Of note, two isolates of SARS-CoV-2 have been identified in Canada and the USA (wild type and D614G mutant) [39]. The D614G mutation involves the RBD, and there is debate as to whether this could affect infectivity or specificity of neutralization. Neutralization assays will therefore be performed for both isolates to allow comparison. These translational studies will inform future use of CCP.

\section{Community engagement}

We established a Community Advisory Committee (CAC) to bring a diversity of perspectives based on relevant lived experiences and socio-demographic characteristics of COVID-19 patients, blood donors, and individuals with specific interests in the pandemic and its impact on the community (e.g. age, sex/gender, education, socioeconomic status, geographic location and race). Advisors from marginalized populations affected by the pandemic will help us understand and advocate for these groups such as residents of long-term care facilities or family members, individuals of colour, and Indigenous people [40,41]. Some of the roles of this committee are (1) to maximize the relevance of the research for the community; (2) to advise on equitable distribution of CCP; (3) to enhance effective dissemination of study results; and (4) to provide feedback on analyses including awareness of the effects of social determinants $[41,42]$. The CAC has seven members and plans to meet monthly.

In summary, the CONCOR-1 trial was designed rapidly as a collaborative effort by a team of experts in clinical trials in collaboration with three blood suppliers. This large randomized trial is adequately powered and sufficiently generalizable to address the efficacy and safety of CCP as potential treatment for COVID-19.

\section{Risks to the trial}

A trial protocol designed to evaluate a new treatment during the ongoing pandemic is subject to risks. For one, enrolment is dependent on disease prevalence; second, the trial requires an adequate supply of plasma which had not been pre-planned; and third, estimates of event rate were based on available data at the outset of the pandemic and may be unstable. To mitigate these risks, we have activated the trial in a large number of centres throughout Canada including both academic and community hospitals, we have expanded into other countries, and we are pursuing methods to combine data with other similar trials without compromising trial integrity. CCP can be shared among the blood suppliers to supply the trial, and we have engaged with the blood suppliers to bolster recruitment of CCP donors. We have built-in a sample size re-estimation halfway through the trial. 


\section{Trial status}

The trial has been approved by Health Canada, the FDA, and the National Research Ethics Commission (Comissão Nacional de Ética em Pesquisa) in Brazil. Research ethics board approvals have been received from all active sites and the blood suppliers. Trial recruitment started on May 8, 2020. There were 52 active sites (48 in Canada, 3 in New York City, 1 in Brazil; an Israeli site was planned but never activated). Over 2000 units of CCP were collected. On January 29, 2021, the IDSMC reviewed the data from the scheduled interim analysis $(n=614)$ and recommended that the trial be stopped for futility. On 29 January 2021, the trial was stopped after 940 patients were enrolled. The currently approved protocol version is 15 Jan 2021 v8.0.

\begin{abstract}
Abbreviations
AE: Adverse event; CAC: Community Advisory Committee; CBS: Canadian Blood Services; CCP: COVID-19 convalescent plasma; CRF: Case report form; CTCAE: Common Terminology Criteria for Adverse Events; EC: Executive Committee; ECMO: Extracorporeal membrane oxygenation; eCRF: Electronic case report form; ELISA: Enzyme-linked immunosorbent assay; FDA: Food and Drug Administration; HQ: Héma-Québec; ICU: Intensive care unit; IDSM C: Independent Data Safety Monitoring Committee; MedDRA: Medical Dictionary for Regulatory Activities; MERS: Middle East respiratory syndrome; NYBC: New York Blood Center; OR: Odds ratio; RBD: Receptor-binding domain; RCT: Randomized controlled trial; REDCap: Research Electronic Data Capture; SAE: Serious adverse event; SARS: Severe acute respiratory syndrome; SC : Steering Committee; SUSAR: Suspected unexpected serious adverse reaction; TACO: Transfusion-associated circulatory overload: TRALI: Transfusion-related acute lung injury
\end{abstract}

\section{Supplementary Information}

Supplementary information accompanies this paper at https://doi.org/10. 1186/s13063-021-05235-3.

Additional file 1. Paper CRF package Version 4.0, 01 Oct 2020.

Additional file 2. Statistical Analysis Plan Version 2.0 19 Mar 2021.

Additional file 3. IDSMC Charter version 2.0, 03 Nov 2020.

Additional file 4. Clinical Trials Ontario Consent Form Template

(English) Version 1.2, 13 Apr 2020 \& Consent Form Template (French- for Quebec sites) Version 1.1, 12 Apr 2020.

\section{Acknowledgements}

The authors wish to acknowledge the contributions of the following research team members: Methods Centre-Melanie St. John, Shannon Lane, Rebecca Pereira, Emily Sirotich; Logistics Centre-Samia Saeed; Québec Coordinating Centre-Julie Morin, Marie Saint-Jacques, Maxime Veillette, Veronica Martinez; NYC Coordinating Centre-Kathleen Kane, Celine Arar, Danielle Hayden, and Lauren Gripp.

Trial Sponsor

Hamilton Health Sciences

1200 Main St W

Hamilton, ON L8N $3 Z 5$

Trial Sponsor Representatives

(United States Only)

Weill Cornell Medical College

1300 York Avenue

New York, NY 10065

(Brazil only)

Fundação Pró-Instituto de Hematologia/Fundarj

8 Frei Caneca Street

Rio de Janeiro, RJ 20211-030

\section{Authors' contributions}

PB, DMA, and JeC conceived the trial. NH, RCo, MPZ, AT, DAF, MMC, MJG, $M C, D V D, N R, R B, N S, A F, A M, D C S, L S, A F T, R Z, N D, L A, C G, M E$, and RCa made substantial contributions to the concept and design of the trial. They drafted and revised the protocol and approved the final version. RCo, DAF, $\mathrm{NL}$, and $\mathrm{YL}$ provided statistical expertise and methodological expertise in clinical trial design. EJ, JuC, JD, KL, CA, AK, DM, MCA, SC, and MA revised the protocol and approved the final version, recruited patients and implemented the trial. LA, BSS, and KCL, along with others, were members of the Steering Committee and provided critical input on study design and execution. $\mathrm{KCL}$ was referred to CONCOR-1 research partnership recruitment by the coordinators of the Strategy for Patient- Oriented Research - Primary and Integrated Health Care Innovations Network. - https://spor-pihci.com/. DMA, JC, PB, NH, and $E J$ wrote the manuscript, and it was reviewed and approved by all authors.

\section{Funding}

Financial support for this trial is provided by: CIHR - COVID-19 May 2020 Rapid Research Funding Opportunity- Operating Grant; Ontario COVID-19 Rapid Research Fund; Toronto COVID-19 Action Initiative 2020 (University of Toronto); University Health Network Emergent Access Innovation Fund; University Health Academic Health Science Centre Alternative Funding Plan (Sunnybrook Health Sciences Centre); Hamilton Academic Health Sciences Organization (HAHSO) Alternative Funding Plan Innovation Grant; Ministère de l'Économie et de l'Innovation (Québec); Saskatchewan Ministry of Health; University of Alberta Hospital Foundation; Sunnybrook Health Sciences Centre Foundation; Fondations CHU Ste-Justine; The Ottawa Hospital Academic Medical Organization; The Ottawa Hospital Foundation COVID-19 Research Fund; Sinai Health System Foundation and McMaster University. The sponsor and funders will have no role in the trial design; collection, management, analysis, and interpretation of data; writing of the report; and the decision to submit the report for publication. The sponsor may review any materials intended for publication but the study SC maintains final authority over the decision to publish.

\section{Availability of data and materials}

Study data may be made available to other investigators upon request. Such requests must first be approved by the Steering Committee. Requests should be sent to arnold@mcmaster.ca.

\section{Declarations}

Ethics approval and consent to participate

All hospital sites had research ethics board approval before trial activation; all participants provided informed consent before any study specific procedures took place. The study was approved by Clinical Trials Ontario (Research Ethics Board of Record: Sunnybrook Health Sciences Centre), project \#2159; the Quebec Ministry of Health and Social Services multicenter ethics review (REB of Record: Comité d'éthique de la recherche du CHU Sainte-Justine), project \#MP-21-2020-2863; Comissão Nacional de Ética em Pesquisa, approval 4.305.792; as well as local research ethics boards of participating hospitals where applicable.

\section{Consent for publication}

Not applicable.

\section{Competing interests}

The authors declare that they have no competing interests.
Author details
${ }^{1}$ Section of Allergy, Immunology and Rheumatology, Department of Pediatrics, CHU Sainte-Justine, Montreal, Quebec, Canada. ${ }^{2}$ Department of Medicine, CHUM, Université de Montréal, Montreal, Quebec, Canada. ${ }^{3}$ Department of Laboratory Medicine and Molecular Diagnostics, Sunnybrook Health Sciences Centre, Toronto, Ontario, Canada. ${ }^{4}$ Department of Laboratory Medicine and Pathobiology, University of Toronto, Toronto, Ontario, Canada. ${ }^{5}$ Department of Medicine, McMaster University, Hamilton, Ontario, Canada. ${ }^{6}$ McMaster Centre for Transfusion Research, McMaster University, Hamilton, Ontario, Canada. ${ }^{7}$ Department of Statistics and Actuarial Science, University of Waterloo, Waterloo, Ontario, Canada. ${ }^{8}$ Department of Medicine, Ottawa Hospital, University of Ottawa, Ottawa, Ontario, Canada. ${ }^{9}$ Ottawa Hospital 
Centre for Transfusion Research, Ottawa Hospital Research Institute, Ottawa, Ontario, Canada. ${ }^{10} \mathrm{C} a n a d i a n$ Blood Services, Ottawa, Ontario, Canada. ${ }^{11}$ Clinical Epidemiology Program, Ottawa Hospital Research Institute, Ottawa, Ontario, Canada. ${ }^{12}$ Department of Medicine, University of Ottawa, Ottawa, Ontario, Canada. ${ }^{13}$ Adjunct Scientist, Canadian Blood Services, Ottawa, Ontario, Canada. ${ }^{14}$ Transfusion Medicine and Cellular Therapy, NewYork-Presbyterian, New York, NY, USA. ${ }^{15}$ Department of Pathology and Laboratory Medicine, Weill Cornell Medicine, New York, NY, USA. ${ }^{16}$ Division of Infectious Diseases, Weill Cornell Medical College, Weill Cornell Medicine, New York, NY, USA. ${ }^{17}$ Department of Medicine (Critical Care), University of Montreal Health Centre (CHUM), Montreal, Quebec, Canada. ${ }^{18}$ Department of Medicine, University of Montreal, Montreal, Quebec, Canada. ${ }^{19}$ Department of Pathology and Laboratory Medicine, University of British Columbia, Ottawa, Ontario, Canada. ${ }^{20}$ Héma-Québec, Saint-Laurent, Montreal, Canada. ${ }^{21}$ Division of Hematology and Oncology, Department of Pediatrics, CHU Sainte-Justine, Université de Montréal, Ottawa, Ontario, Canada. ${ }^{22}$ Medical Affairs and Innovation, Héma-Québec, Saint-Laurent, Montreal, Canada. ${ }^{23}$ Departments of Medicine, Laboratory Medicine and Pathobiology, Institute of Health Policy Management and Evaluation, University of Toronto, Toronto, Ontario, Canada. ${ }^{24}$ Division of Hematology, Mount Sinai Hospital, Toronto, Ontario, Canada. ${ }^{25}$ Canadian Blood Services, Toronto, Ontario, Canada. ${ }^{26}$ Département de Microbiologie, Infectiologie et Immunologie, Université de Montréal, Montreal, Quebec, Canada. ${ }^{27} \mathrm{CHUM}$ Research Center, Montreal, Quebec, Canada. ${ }^{28}$ Department of Microbiology, Sinai Health System, Toronto, Ontario, Canada. ${ }^{29}$ Department of Laboratory Medicine and Pathobiology and Dalla Lana School of Public Health, University of Toronto, Toronto, Ontario, Canada. ${ }^{30}$ Department of Critical Care Medicine, Sunnybrook Health Sciences Centre, Toronto, Ontario, Canada. ${ }^{31}$ Department of Medicine, Interdepartmental Division of Critical Care, University of Toronto, Toronto, Ontario, Canada. ${ }^{32}$ Department of Health Research Methods, Evidence \& Impact, Faculty of Health Sciences, McMaster University, Hamilton, Ontario, Canada. ${ }^{33}$ Department of Anesthesiology and Critical Care Medicine, Division of Critical Care Medicine, Faculty of Medicine, Université Laval, Quebec, Quebec, Canada. ${ }^{34} \mathrm{CHU}$ de Québec - Université Laval Research Centre, Population Health and Optimal Health Practices Research Unit, Trauma Emergency - Critical Care Medicine, Université Laval, Quebec, Quebec, Canada. ${ }^{35}$ Department of Internal Medicine, Sections of Hematology/Medical Oncology and Critical Care, University of Manitoba, Winnipeg, Manitoba, Canada. ${ }^{36}$ Department of Medicine, Division of Infectious Diseases, Sunnybrook Health Sciences Centre, University of Toronto, Toronto, Ontario, Canada. ${ }^{37}$ Hemorio, Rio de Janeiro, Brazil. ${ }^{38}$ Hematology Institute and Blood Bank, Meir Medical Center, Tel Aviv, Israël. ${ }^{39}$ Sackler School of Medicine, Tel Aviv University, Tel Aviv, Israël. ${ }^{40}$ New York Blood Center Enterprises, New York, NY, USA. ${ }^{41}$ Department of Computing and Software, McMaster University, Hamilton, Ontario, Canada. ${ }^{42}$ Department of Community Health Sciences, University of Calgary, Hamilton, Ontario, Canada. ${ }^{43}$ Clinical Research Department, Centre de recherche du CHU Sainte-Justine, Centre Hospitalier Universitaire Sainte-Justine Centre, Montreal, Canada.

\section{Received: 12 January 2021 Accepted: 29 March 2021} Published online: 04 May 2021

\section{References}

1. Worldometers. COVID-19 Coronovirus Pandemic. https://www.worldometers. info/coronavirus/. Accessed 18 May 2020.

2. Casadevall A, Pirofski L. The convalescent sera option for containing COVID19. J Clin Invest. 2020;130(4):1545-8. https://doi.org/10.1172/JCl138003.

3. Shen C, Wang Z, Zhao F, Yang Y, Li J, Yuan J, Wang F, Li D, Yang M, Xing L, Wei J, Xiao H, Yang Y, Qu J, Qing L, Chen L, Xu Z, Peng L, Li Y, Zheng H, Chen F, Huang K, Jiang Y, Liu D, Zhang Z, Liu Y, Liu L. Treatment of 5 critically ill patients with COVID-19 with convalescent plasma. JAMA. 2020; 323(16):1582-9. https://doi.org/10.1001/jama.2020.4783.

4. Cheng Y, Wong R, Soo YOY, Wong WS, Lee CK, Ng MHL, Chan P, Wong KC, Leung CB, Cheng $\mathrm{G}$. Use of convalescent plasma therapy in SARS patients in Hong Kong. Eur J Clin Microbiol Infect Dis. 2005;24(1):44-6. https://doi. org/10.1007/s10096-004-1271-9.

5. Hung IFN, To KKW, Lee CK, Lee KL, Chan K, Yan WW, et al. Convalescent plasma treatment reduced mortality in patients with severe pandemic influenza A (H1N1) 2009 virus infection. Clin Infect Dis. 2011;52(4):447-56. https://doi.org/10.1093/cid/ciq106.
6. Beigel JH, Aga E, Elie-Turenne MC, Cho J, Tebas P, Clark CL, Metcalf JP, Ozment C, Raviprakash K, Beeler J, Holley HP Jr, War1ner S, Chorley C, Lane HC, Hughes MD, Davey RT Jr, Beigel JH, Aga E, Elie-Turenne MC, Cho J, Tebas P, Clark CL, Metcalf JP, Ozment C, Raviprakash K, Beeler J, Holley HP Jr, Warner S, Chorley C, Lane HC, Hughes MD, Davey RT, Barron M, Bastani A, Bauer P, Borkowsky W, Cairns C, Deville J, Elie MC, Fichtenbaum C, Finberg R, Jain M, Kaufman D, Lin M, Lin J, Maves R, Morrow L, Nguyen MH, Park P, Polk C, Randolph A, Rao S, Rubinson L, Schofield C, Shoham S, Stalets $\mathrm{E}$, Stapleton RD. Anti-influenza immune plasma for the treatment of patients with severe influenza $\mathrm{A}$ : a randomised, double-blind, phase 3 trial. Lancet Respir Med. 2019;7(11):941-50. https://doi.org/10.1016/S2213-2600(1 9)30199-7.

7. Sahr F, Ansumana R, Massaquoi TA, Idriss BR, Sesay FR, Lamin JM, Baker $S$, Nicol S, Conton B, Johnson W, Abiri OT, Kargbo O, Kamara P, Goba A, Russell JB, Gevao SM. Evaluation of convalescent whole blood for treating Ebola virus disease in Freetown, Sierra Leone. J Inf Secur. 2017;74(3):302-9. https://doi.org/10.1016/j.jinf.2016.11.009.

8. Van Griensven J, Edwards T, De Lamballerie X, Semple MG, Gallian P, Baize $\mathrm{S}$, et al. Evaluation of convalescent plasma for Ebola virus disease in Guinea. N Engl J Med. 2016;374(1):33-42. https://doi.org/10.1056/NEJMoa1511812.

9. Mair-Jenkins J, Saavedra-Campos M, Baillie JK, Cleary P, Khaw FM, Lim WS, Makki S, Rooney KD, Convalescent Plasma Study Group, Nguyen-van-Tam $J S$, Beck CR. The effectiveness of convalescent plasma and hyperimmune immunoglobulin for the treatment of severe acute respiratory infections of viral etiology: a systematic review and exploratory meta-analysis. J Infect Dis. 2015;211(1):80-90. https://doi.org/10.1093/infdis/jiu396.

10. Devasenapathy N, Ye Z, Loeb M, Fang F, Najafabadi B, Xiao Y, et al. Indirect evidence on efficacy and safety of convalescent plasma in severe COVID-19 patients: systematic review and meta-analysis. CMAJ. 2020;192(27):E745-55. https://doi.org/10.1503/cmaj.200642.

11. Duan K, Liu B, Li C, Zhang H, Yu T, Qu J, Zhou M, Chen L, Meng S, Hu Y, Peng C, Yuan M, Huang J, Wang Z, Yu J, Gao X, Wang D, Yu X, Li L, Zhang J, Wu X, Li B, Xu Y, Chen W, Peng Y, Hu Y, Lin L, Liu X, Huang S, Zhou Z, Zhang L, Wang Y, Zhang Z, Deng K, Xia Z, Gong Q, Zhang W, Zheng X, Liu Y, Yang H, Zhou D, Yu D, Hou J, Shi Z, Chen S, Chen Z, Zhang X, Yang X. Effectiveness of convalescent plasma therapy in severe COVID-19 patients. Proc Natl Acad Sci. 2020;117(17):9490-6. https://doi.org/10.1073/pnas.20041 68117.

12. Zhang B, Liu S, Tan T, Huang W, Dong Y, Chen L, Chen Q, Zhang L, Zhong Q, Zhang X, Zou Y, Zhang S. Treatment with convalescent plasma for critically ill patients with SARS-CoV-2 infection. Chest. 2020;158(1):e9-13. https://doi.org/10.1016/j.chest.2020.03.039.

13. Zeng Q, Yu Z, Gou J, Li G, Ma S, Zhang G, et al. Effect of convalescent plasma therapy on viral shedding and survival in COVID-19 patients. J Infect Dis. 2020;222(1):38-43. https://doi.org/10.1093/infdis/jiaa228.

14. Li L, Zhang W, Hu Y, Tong X, Zheng S, Yang J, Kong Y, Ren L, Wei Q, Mei H, Hu C, Tao C, Yang R, Wang J, Yu Y, Guo Y, Wu X, Xu Z, Zeng L, Xiong N, Chen L, Wang J, Man N, Liu Y, Xu H, Deng E, Zhang X, Li C, Wang C, Su S, Zhang L, Wang J, Wu Y, Liu Z. Effect of convalescent plasma therapy on time to clinical improvement in patients with severe and life-threatening COVID-19: a randomized clinical trial. JAMA. 2020;324(5):460-70. https://doi. org/10.1001/jama.2020.10044.

15. Gharbharan A, Jordans CCE, GeurtsvanKessel C, den Hollander JG, Karim F, Mollema FPN, et al. Convalescent plasma for COVID-19. A randomized clinical trial. medRxiv. 2020; https://doi.org/10.1101/2020.07.01.20139857.

16. Agarwal A, Mukherjee A, Kumar G, Chatterjee P, Bhatnagar T. Convalescent plasma in the management of moderate COVID-19 in India: an open-label parallel-arm phase II multicentre randomized controlled trial (PLACID trial). BMJ. 2020;371:m3939.

17. Tanne JH. Covid-19: FDA approves use of convalescent plasma to treat critically ill patients. BMJ. 2020;368:m1256.

18. Joyner MJ, Senefeld JW, Klassen SA, Mills JR, Johnson PW, Theel ES, et al. Effect of convalescent plasma on mortality among hospitalized patients with COVID-19: initial three month experience. medRxiv. 2020; https://doi. org/10.1101/2020.08.12.20169359.

19. Joyner MJ, Wright RS, Fairweather D, Senefeld JW, Bruno KA, Klassen SA, Carter RE, Klompas AM, Wiggins CC, Shepherd JRA, Rea RF, Whelan ER, Clayburn AJ, Spiegel MR, Johnson PW, Lesser ER, Baker SE, Larson KF, Ripoll JG, Andersen KJ, Hodge DO, Kunze KL, Buras MR, Vogt MNP, Herasevich V, Dennis JJ, Regimbal RJ, Bauer PR, Blair JE, van Buskirk CM, Winters JL, Stubbs JR, Paneth NS, Verdun NC, Marks P, Casadevall A. Early safety indicators of 
COVID-19 convalescent plasma in 5000 patients. J Clin Invest. 2020;130(9): 4791-7. https://doi.org/10.1172/JCl140200.

20. Joyner MJ, Bruno KA, Klassen SA, Kunze KL, Johnson PW, Lesser ER, Wiggins CC, Senefeld JW, Klompas AM, Hodge DO, Shepherd JRA, Rea RF, Whelan ER, Clayburn AJ, Spiegel MR, Baker SE, Larson KF, Ripoll JG, Andersen KJ, Buras MR, Vogt MNP, Herasevich V, Dennis JJ, Regimbal RJ, Bauer PR, Blair JE, van Buskirk CM, Winters JL, Stubbs JR, van Helmond N, Butterfield BP, Sexton MA, Diaz Soto JC, Paneth NS, Verdun NC, Marks P, Casadevall A, Fairweather DL, Carter RE, Wright RS. Safety update: COVID-19 convalescent plasma in 20,000 hospitalized patients. Mayo Clin Proc. 2020;95(9):1888-97. https://doi.org/10.1016/.mayocp.2020.06.028.

21. Liu L, Wei Q, Lin Q, Fang J, Wang H, Kwok H, Tang H, Nishiura K, Peng J, Tan Z, Wu T, Cheung KW, Chan KH, Alvarez X, Qin C, Lackner A, Perlman S, Yuen $\mathrm{KY}$, Chen $Z$. Anti-spike IgG causes severe acute lung injury by skewing macrophage responses during acute SARS-CoV infection. JCl Insight. 2019; 4(4):e123158. https://doi.org/10.1172/jci.insight.123158.

22. Yeh K, Chiueh T, Siu LK, Lin J, Chan PKS, Peng M, et al. Experience of using convalescent plasma for severe acute respiratory syndrome among healthcare workers in a Taiwan hospital. J Antimicrob Chemother. 2005; 56(5):919-22. https://doi.org/10.1093/jac/dki346.

23. Beigel JH, Tebas P, Elie-Turenne MC, Bajwa E, Bell TE, Cairns CB, Shoham S, Deville JG, Feucht E, Feinberg J, Luke T, Raviprakash K, Danko J, O'Neil D, Metcalf JA, King K, Burgess TH, Aga E, Lane HC, Hughes MD, Davey RT, Tebas P, Quinn J, Jiang Y, Elie-Turenne MC, Hoelle R, lovine N, Wills RS, Pata S, Huggins M, Manukian B, Bajwa E, Holland C, Brait K, Hunt T, Stowell C, Slater A, Bell TE, Townsends M, Cairns CB, Quackenbush EB, Park YA, Jordan PG, Blanchet C, Chronowski K, Alvarez K, Shoham S, Ostrander D, Woessner T, Thoman S, Deville JG, Lin J, Ziman A, Shankar K, Feucht E, Blok T, Batts D, Beck B, Massey G, Bradley C, Feinberg J, Carey P, Baer J, Whitehead EM, Kohrs S, Giulitto R, Schofield C, Fairchok M, Chambers S, Baker C, RN, Parker M, Harshbarger M, Nguyen MH, Carey ME, Paronish J, Cornell F, Cramer J, Pakstis DL, Ison MG, Wunderink R, Glesby M, Ham K, Hughes V, Cushing M, Goss C, Grenade J, Park PK, Napolitano LM, Raghavendran K, Hyzy RC, Davenport R, Brierley K, Downs T, Gong MN, Uehlinger J, Lin M, Fritsche J, Green T, McLeod B, Patel D, Bavaro MF, Deiss R, Brandt C, Cammarata S, Kremp A, Hollis-Perry K, Lalani T, Banks S, Johnson J, Maguire J, McNiff J, Rigg LE, Ganesan A, Barahona I, Danko J, Spencer S, Stagliano D, Burgess T, Talmor D, Mohammed M, Banner-Goodspeed V, Salata R, Finberg R, Wang J, Longtine K, Longtine J, O'Neil M, Bauer PR, Gajic O, Weist SM, Sevransky J, Brown M, Roback J, Oropello J, Twohig B, Jhang J, Seethala R, Chen WH, Fontaine M, Saharia K, Husson J, DeBiasi R, Wilson JL, Criss VR, Voell J, Leitman S, Atkins JW, Patel H, Paige T, Cantilena C, Siegel D, DeMuth F, Fletcher $\mathrm{CH}$, Pelletier JPR, Alnuaimat $\mathrm{H}$, Pourde M. Immune plasma for the treatment of severe influenza: an open-label, multicentre, phase 2 randomised study. Lancet Respir Med. 2017;5(6):500-11. https://doi.org/10.1 016/S2213-2600(17)30174-1.

24. Davey RT, Fernández-Cruz E, Markowitz N, Pett S, Babiker AG, Wentworth D, et al. Anti-influenza hyperimmune intravenous immunoglobulin for adults with influenza A or B infection (FLU-IVIG): a double-blind, randomised, placebo-controlled trial. Lancet Respir Med. 2019;7(11):951-63. https://doi. org/10.1016/S2213-2600(19)30253-X.

25. World Health Organization. Clinical management Clinical management: Living guidance COVID-19. https:/www.who.int/publications//item/WHO-2 019-nCoV-clinical-2021-1. Accessed 10 Mar 2021.

26. Medical Dictionary for Regulatory Activities. Welcome to MedDRA. https:// www.meddra.org/. Accessed 12 Jun 2020

27. National Cancer Institute. Common Terminology Criteria for Adverse Events (CTCAE). https://ctep.cancer.gov/protocolDevelopment/electronic_applica tions/ctc.htm Accessed 12 Jun 2020.

28. International Society of Blood Transfusion Working Party on Haemovigilance. Proposed Standard Definitions for Surveillance of Non Infectious Adverse Transfusion Reactions. http://www.isbtweb.org/fileadmin/ user_upload/files-2015/haemovigilance/definitions/Proposed/definitions/2 011/surveillance/non/infectious/adverse/reactions/haemovigilance/incl/TRA Ll/correction/2013.pdf. Accessed 12 Jun 2020.

29. Cook D, Lauzier F, Rocha MG, Sayles MJ, Finfer S. Serious adverse events in academic critical care research. CMAJ. 2008;178(9):1181-4. https://doi.org/1 0.1503/cmaj.071366.

30. Herdman M, Gudex C, Lloyd A, Janssen M, Kind P, Parkin D, Bonsel G, Badia $X$. Development and preliminary testing of the new five-level version of EQ-
5D (EQ-5D-5L). Qual Life Res. 2011;20(10):1727-36. https://doi.org/10.1007/ S11136-011-9903-X.

31. Harris PA, Taylor R, Thielke R, Payne J, Gonzalez N, Conde JG. Research electronic data capture (REDCap)-a metadata-driven methodology and workflow process for providing translational research informatics support. J Biomed Inform. 2009;42(2):377-81. https://doi.org/10.1016/j.jbi.2008.08.010.

32. Harris PA, Taylor R, Minor BL, Elliott V, Fernandez M, O'Neal L, et al. The REDCap consortium: building an international community of software platform partners. J Biomed Inform. 2019;95:103208.

33. Public Health Agency of Canada. Coronavirus Disease 2019 (COVID-19). https://www.canada.ca/content/dam/phac-aspc/documents/services/disea ses/2019-novel-coronavirus-infection/surv-covid19-epi-update-eng.pdf. Accessed 09 May 2020.

34. Richardson S, Hirsch JS, Narasimhan M, Crawford JM, McGinn T, Davidson KW, and the Northwell COVID-19 Research Consortium, Barnaby DP, Becker LB, Chelico JD, Cohen SL, Cookingham J, Coppa K, Diefenbach MA, Dominello AJ, Duer-Hefele J, Falzon L, Gitlin J, Hajizadeh N, Harvin TG, Hirschwerk DA, Kim EJ, Kozel ZM, Marrast LM, Mogavero JN, Osorio GA, Qiu M, Zanos TP. Presenting characteristics, comorbidities, and outcomes among 5700 patients hospitalized with COVID-19 in the New York City area. JAMA. 2020;323(20):2052-9. https://doi.org/10.1001/jama.2020.6775.

35. Lan K, DeMets D. Discrete sequential boundaries for clinical trials. Biometrika. 1983;70(3):659-63. https://doi.org/10.2307/2336502.

36. O'Brien P, Fleming T. A multiple testing procedure for clinical trials. Biometrics. 1979;35(3):549-56. https://doi.org/10.2307/2530245.

37. Liu STH, Lin HM, Baine I, Wajnberg A, Gumprecht JP, Rahman F, Rodriguez D, Tandon P, Bassily-Marcus A, Bander J, Sanky C, Dupper A, Zheng A, Nguyen FT, Amanat F, Stadlbauer D, Altman DR, Chen BK, Krammer F, Mendu DR, Firpo-Betancourt A, Levin MA, Bagiella E, Casadevall A, CordonCardo C, Jhang JS, Arinsburg SA, Reich DL, Aberg JA, Bouvier NM. Convalescent plasma treatment of severe COVID-19: a propensity scorematched control study. Nat Med. 2020;26(11):1708-13. https://doi.org/10.103 8/541591-020-1088-9.

38. Food and Drug Administration. Investigational COVID-19 Convalescent Plasma Guidance for Industry. https:/www.fda.gov/media/136798/downloa d. Accessed 12 Jun 2020.

39. Korber B, Fischer WM, Gnanakaran S, Yoon H, Theiler J, Abfalterer W, Hengartner N, Giorgi EE, Bhattacharya T, Foley B, Hastie KM, Parker MD, Partridge DG, Evans CM, Freeman TM, de Silva TI, McDanal C, Perez LG, Tang H, Moon-Walker A, Whelan SP, LaBranche CC, Saphire EO, Montefiori DC, Angyal A, Brown RL, Carrilero L, Green LR, Groves DC, Johnson KJ, Keeley AJ, Lindsey BB, Parsons PJ, Raza M, Rowland-Jones S, Smith N, Tucker RM, Wang D, Wyles MD. Tracking changes in SARS-CoV-2 spike: evidence that D614G increases infectivity of the COVID-19 virus. Cell. 2020;182(4):81227. https://doi.org/10.1016/j.cell.2020.06.043.

40. Ahmed SM, Palermo AGS. Community engagement in research: frameworks for education and peer review. Am J Public Health. 2010;100(8):1380-7. https://doi.org/10.2105/AJPH.2009.178137.

41. World Health Organisation. RCCE Action Plan Guidance Covid-19 preparedness \& response. https://www.who.int/publications-detail/riskcommunication-and-community-engagement-(rcce)-action-plan-guidance. Accessed 12 Jun 2020

42. Holzer JK, Ellis L, Merritt MW. Why we need community engagement in medical research. J Investig Med. 2014;62(6):851-5. https://doi.org/10.1097/ JIM.0000000000000097.

\section{Publisher's Note}

Springer Nature remains neutral with regard to jurisdictional claims in published maps and institutional affiliations. 